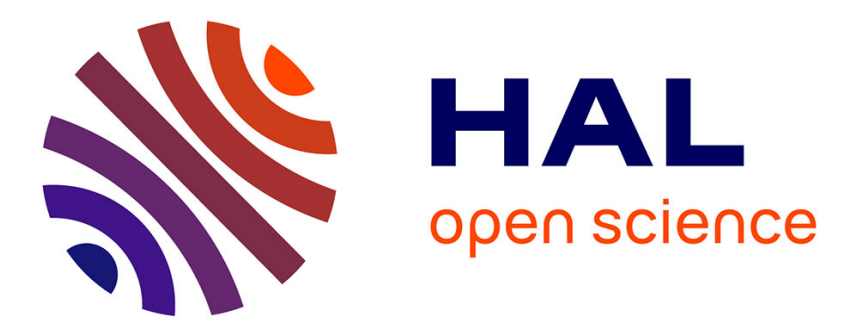

\title{
Link-Layer Based Anycast Routing for a Dynamic and Distributed Portal Selection in Wireless Mesh Networks
}

A. Ariza-Quintana, A. Triviño-Cabrera, E. Casilari

\section{To cite this version:}

A. Ariza-Quintana, A. Triviño-Cabrera, E. Casilari. Link-Layer Based Anycast Routing for a Dynamic and Distributed Portal Selection in Wireless Mesh Networks. 9th Wired/Wireless Internet Communications (WWIC), Jun 2011, Vilanova i la Geltrú, Spain. pp.374-385, 10.1007/978-3-642-21560-5_31 . hal-01583642

\section{HAL Id: hal-01583642 \\ https://hal.inria.fr/hal-01583642}

Submitted on 7 Sep 2017

HAL is a multi-disciplinary open access archive for the deposit and dissemination of scientific research documents, whether they are published or not. The documents may come from teaching and research institutions in France or abroad, or from public or private research centers.
L'archive ouverte pluridisciplinaire HAL, est destinée au dépôt et à la diffusion de documents scientifiques de niveau recherche, publiés ou non, émanant des établissements d'enseignement et de recherche français ou étrangers, des laboratoires publics ou privés. 


\title{
Link-Layer Based Anycast Routing for a Dynamic and Distributed Portal Selection in Wireless Mesh Networks
}

\author{
A. Ariza-Quintana, A. Triviño-Cabrera, E. Casilari \\ Dpto. Tecnología Electrónica, E.T.S.I. Telecomunicación, University of Málaga \\ Campus Teatinos \\ 29071 Málaga, Spain \\ \{atc, aarizaq, ecasilari\}@uma.es
}

\begin{abstract}
Wireless mesh networks may be equipped with multiple Portals that offer access to external networks such as the Internet. These Portals are provided with two interfaces: one to a wired network (to communicate with other Portals and Gateways) and another one to the wireless network. In the current $802.11 \mathrm{~s}$ standard, the interaction between these two interfaces is not defined. By connecting both interfaces in a seamless way, this paper addresses and solves the problem of dynamically selecting multiple Portals in a Wireless Mesh Network. In particular, the proposal is supported by the definition of an anycast group formed by the Portals in the network. Any data frame whose destination is an external host is routed to any Portal in the network. The route is selected depending on the instantaneous network conditions (e.g. path availability, number of hops, delay or losses). To make this selection a distributed process, a link-layer based forwarding scheme is employed. By means of simulations, we state that the application of the proposed technique yields to significant improvements concerning the network performance.
\end{abstract}

Keywords: Mesh Portal, Wireless mesh network, Multiple Mesh Portal, Anycasting.

\section{Introduction}

Wireless communications have become a common mechanism to connect machines. This expansion of wireless technologies has prompted the more popular mobile devices (smartphones, electronic pads, tablets and laptops) to be equipped with one or multiple built-in radio interfaces: one interface to connect to a telecommunication operator and another one to communicate in the free unlicensed spectrum. In order to facilitate their integration into the Internet in a cost-effective way, some cities, organisms and business have opted for the development of their own wireless mesh network [1] [2]. Due to this popularity, IEEE 802.11s has been released as an experimental standard amendment for wireless mesh networks [3]. According to these specifications, a wireless mesh network is formed by a set of dedicated, static nodes which are wirelessly interconnected leading to a multihop backbone [4]. These nodes, which are called Mesh Points (MP), act as static relays for other mesh nodes by executing a link layer based routing protocol called HWMP (Hybrid Wireless Mesh Protocol) [5]. However, some MPs offer additional features. In this sense, the Mesh 
Access Points (MAP) provide access to mobile devices or stations (STA). On the other hand, the Mesh Portals (MPP) are MPs which are outfitted with an Internet connection. MPPs fulfill a similar role to a gateway in a typical IP-based access network. In order to communicate with the Internet, the STA or mobile client (user) has to connect to any MAP in the backbone. Then, the MAP discovers and selects the best path (composed of a sequence of MPs) to route the messages to a certain MPP.

In a wireless mesh network with multiple MPPs, the selection of the MPP to be used by the traffic flowing from/to a STA can impact on the network performance. In this sense, some policies have already been published for $802.11 \mathrm{~s}$ based and IP-based access networks. In order to describe the particularities of the existing proposals in a generic way, MPPs and Gateways will be called connection nodes. The connection nodes are provided with different functionalities which depend on the proposals. Basically, a scheme in this context can be characterized by the five following features: (i) how the connection node is discovered, (ii) whether the discovery is supported by an anycast transmission, (iii) the capability to use multiple connection nodes simultaneously, (iv) the selection of the connection node and (v) whether the connection nodes collaborate in the intra-mesh routing (i.e. the routing between two MPs belonging to the same wireless mesh network). Concerning the anycast transmission, the related works restrict this capability to the discovery of the connection nodes. Once they are identified, the data transmission is forwarded to a fixed destination node. This setting prevents the flexibility of having multiple connection nodes from being fully exploited so that the instantaneous radio characteristics could be also taken into account in the data transmissions. In order to overcome this limitation, we propose that the MAC addresses of the MPPs can be interchangeable in both phases (discovery and transmission). Moreover, we have incorporated the capability of benefiting from the wired connection among the MPPs for the intra-mesh traffic. We have conducted extensive simulations in OMNeT++ [6] and the results confirm the performance enhancements of our proposal.

The rest of the paper is organized as follows. Section 2 describes the related work proposed in this area. Section 3 details the proposal. The proposed algorithm is evaluated in Section 4. Finally, Section 5 draws the main conclusions of our work.

\section{Related Work}

Several research works analyze the use of multiple connection nodes (a MPP in a 802.11s based network or a Gateway in an IP-based access network) in a wireless mesh network. These proposals can be classified according to the following issues:

- Timing of the procedures to discover the connection nodes. This characteristic refers to the instant when the procedures to discover the connection nodes are triggered. In this sense, the proposals can be divided into reactive, proactive or hybrid. Under the reactive or on demand mechanism, a node willing to get access to the Internet emits a message to become aware of the routes to the connection nodes. The message is broadcast to the condition that the source does not keep in their routing tables any information about a path to the connection nodes or when this information is assumed to be stale. Examples of 
this kind of implementation can be found in [7] [8]. On the other hand, the proactive schemes are accomplished by the periodic emission of announcements generated by the connection nodes. The sources receiving the messages acquire the routing information to transmit their data to the external hosts. Some examples of this kind of schemes are described in [9] [10]. Finally, in the hybrid schemes, the periodic announcements generated by the connection nodes are propagated to an area around them and defined by the maximum number of times (hops) that the messages can be retransmitted. The nodes outside this area employ a reactive approach. Authors in [11] present a hybrid scheme. There also exist some hybrid approaches that employ the proactive or the reactive mechanism depending on the functionality of the nodes. HWMP (Hybrid Hybrid Wireless Mesh Protocol) defines two alternatives to establish the paths: one for intra-mesh traffic and another one for the external (Internet) traffic [5]. For the Internet traffic, spanning trees rooted at the MPP are periodically updated. Towards this goal, the MPP emits Root Announcements (RANN). The work in [12] proposes to selectively retransmit the RANN in order to allow the use of multiple MPP in the network. An enhanced version of HWMP is presented in [13] to support Quality of Service.

- Capability to perform the discovery of the connection nodes by an anycast transmission. Some proposals define an anycast group to which the connection nodes attach. Then, the discovery is supported by messages to the anycast group. In IP-based access networks, this capability is incorporated in [7] [11]. On the other hand, the 'One Laptop Per Child' (OLPC) networking solution proposes MPPs to be discovered by means of a link layer anycast MAC address [14]. This discovery is mainly prompted to simplify the HWMP by avoiding its proactive behavior.

- Capability to associate to multiple connection nodes. When multiple connection nodes are available, some research works suggest that Internet flows use them simultaneously. A multipath routing protocol may support this capability so this kind of schemes is often termed as multi-gateway, multipath or multiple-association. Works in [8] and [12] are examples of proposals holding this capability.

- Selection of the connection node. Once that the reachable connection nodes are discovered, a decision about which one to employ is required. The decision may consider diverse parameters such as the radio link rate, the Gateway load [15] or the number of hops [16]. For instance, a novel metric which combines the gateway load, the route interference and the link quality is presented in [9]. Alternatively, the work in [17] proposes a mathematical formulation to enable the construction of a Spanning Tree rooted at the Gateway. In this way, the paths from any node to the Gateway are composed of nodes which have selected the same Gateway as the best one. The selection of the connection node could also affect the retransmission of the announcements generated by these Internet-connected nodes as portrayed in [12]. 
- Participation of the connection nodes in the intra-mesh routing. In some schemes, the intra-mesh routing benefit from the routing information acquired by the connection nodes. In [12], the MPPs also collaborate in the discovery of paths among nodes in the same network. With this intention, in order to create a route, a MP sends the frames targeted to another MP towards the MPP, which retransmits them to the intended MP. Once the first frame is received by the final MP, it triggers a mechanism to set a bidirectional path to the source.

\section{Link-Layer Based Anycast Routing for 802.11s networks}

Our proposal is intended for wireless networks where routing procedures are executed at the link layer. In this kind of networks, $802.11 \mathrm{~s}$ based networks outstand. The proposal provides three fundamental features. Firstly, the MPPs are discovered by means of an anycast-like scheme. Moreover, this anycast-like procedure is not restricted to the MPP discovery but it is also employed in the data transmission. An additional contribution of this work relies on the fact that the routing information for both interfaces (wired and wireless) is combined in the MPPs. In this way, the communication between MPs could take advantage of the wired connection among the MPPs. The details about these characteristics are described next.

\subsection{Anycast-like discovery of the MPPs}

In the link layer addressing scheme, an anycast MAC address is not defined. OLPC proposes to set a particular MAC address as an anycast address [14]. The problem of this approach is that all the MPs in the network should be configured to properly work with this address. In order to avoid this configuration in all the MPs, we propose a novel mechanism that emulates the anycast discovery in $802.11 \mathrm{~s}$ networks. The mechanism is supported by the proactive discovery of the MPPs. In this sense, a MPP becomes aware of all the mesh nodes acting as MPPs through the wired network. One of them is periodically selected to 'represent' the group. The representation involves the emission of periodic messages in the wireless network. This specific message informs about all the available MPPs in the wireless mesh network. Alternatively, the MPs may trigger the procedures to discover the MPP when its corresponding routing information has become stale. In this way, the MP node emits a message in the neighborhood so that a directly-connected node can respond to it with the information associated to the MPPs. This mechanism is usually performed when a new node enters the network.

Upon reception of the MPP announcements, a MP becomes aware of the MAC addresses of the MPPs in the network. The anycast transmission is emulated in this work by setting the MPP MAC addresses as interchangeable. With this goal, the management of the interchangeable addresses is supported by a data structure called Equivalent Address Table (EAT). This structure is created and maintained by the nodes. 
In this phase, the routing information is not completed. A routing protocol needs to be executed to determine the communication costs to every MPP. A routing metric to assess the communication cost will be used to decide about the preferable MPP to which the frames must be transmitted. The present approach does not require any specific communication cost to decide about the suitability of an MPP to be used. In our experiments, we have opted for the number of hops as the route metric.

One of the main advantages of the proposal is that it can be gradually incorporated to the mesh network as it can be executed even when there exist some mesh nodes that do not implement it. These MPs will ignore the additional information contained in the MPP announcements and, in turn, will not be able to work with the EAT. An additional pro of the presented approach relies on the fact that the Equivalent Address Table could be also employed for other kind of groups or clusters. Thus, the technique provides a flexible mechanism to emulate the anycast transmission for any group in the network.

\subsection{Anycast-like data forwarding}

Once the EAT is configured, the anycast support is extended to the frame retransmissions. Although the source sets the preferable MPP in the destination field of the frame, a relaying node in the route to this MPP may decide that an alternative MPP is more appropriate for the transmission. This is the case of a relaying node that has detected that the MPP set in the destination field has become unreachable or when the forwarding node has updated the communication costs, which make the use of another MPP to be more cost-effective. Under these circumstances, the relaying node changes in the frame the destination MPP. The change is supported by the EAT and the routing table. Fig. 1 illustrates an example about how this procedure works. The dashed lines represent wireless links whereas the solid lines symbolize the wired connections. In this case, MP-A generates a frame to the Internet. To make a routing decision, MP-A looks up at its routing table and decides that the best MPP to send the frames is MPP3. According to this routing entry, MP-A retransmits the frame to MP$\mathrm{B}$. The destination field of the frame is set to MPP3 by the node MP-A. When MP-B receives the frame, it detects that the destination defined in the corresponding field is included in its EAT. Thus, it is an interchangeable address. MP-B evaluates the convenience of continuing sending the frame to MPP3. With this purpose, it analyzes the routing entries of those addresses included in its EAT. The communications costs perceived by MP-B reveal that transmitting the frame to MPP2 is more convenient since the cost (in this case, set in terms of number of hops) to MPP2 is 1 whereas the cost to MPP3 is 3 . Under these circumstances, MP-B decides to change the MAC address of the destination field to MPP2. Consequently, MP-B will retransmit the frame according to the routing information related to the MPP2 entry.

The retransmission based on the EAT is able to consider the instantaneous communication costs (path availability, number of hops, losses, delay or any valid metric) associated to the alternative nodes and the current destination. Furthermore, a dynamic selection of the routing path is established for every frame. The MP can use multiple MPPs so the scheme allows a multi-association. Additionally, the use of 
interchangeable addresses is also employed to forward control frames such as Route Requests.

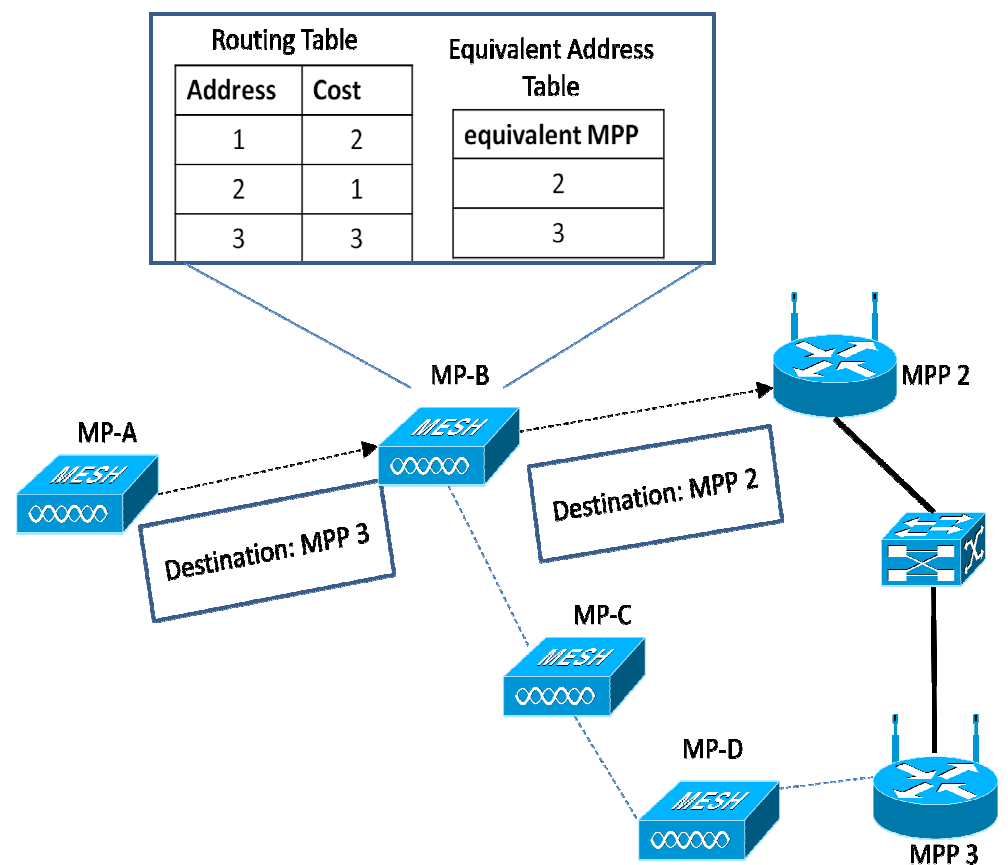

Fig. 1. Illustration of the anycast-like retransmission of a frame. A redirection occurs in MP-B.

\subsection{Combination of the routing information in the MPPs}

Mesh Portals (MPPs) are usually connected by means of a high-rate wired infrastructure such as a Gigabit-Ethernet or a FastGigabitEthernet. Thus, MPPs are provided with two interfaces: one for the Ethernet and another one for the wireless mesh network. In our proposal, these two modules are connected so that the routing information in both networks is combined.

By combining both types of information, the MPPs could participate in the routing between two MPs belonging to the same wireless mesh network. Conversely to other previous proposals, the collaboration of the MPPs is not restricted to the route discovery but MPPs may also participate in the retransmission of the frames. Fig. 2 shows how this new functionality works. In this case, MP-A needs to transmit a frame to MP-B. MP-A is not directly connected to MP-B while the links in the wireless mesh network do not offer any route to connect the nodes. The unique (and optimal) route is composed of some MPPs. Since MP-A is connected to a MPP, the selected route uses the wired infrastructure to transmit the frame to the destination. The arrows represent the sequence of retransmissions for the frame. 


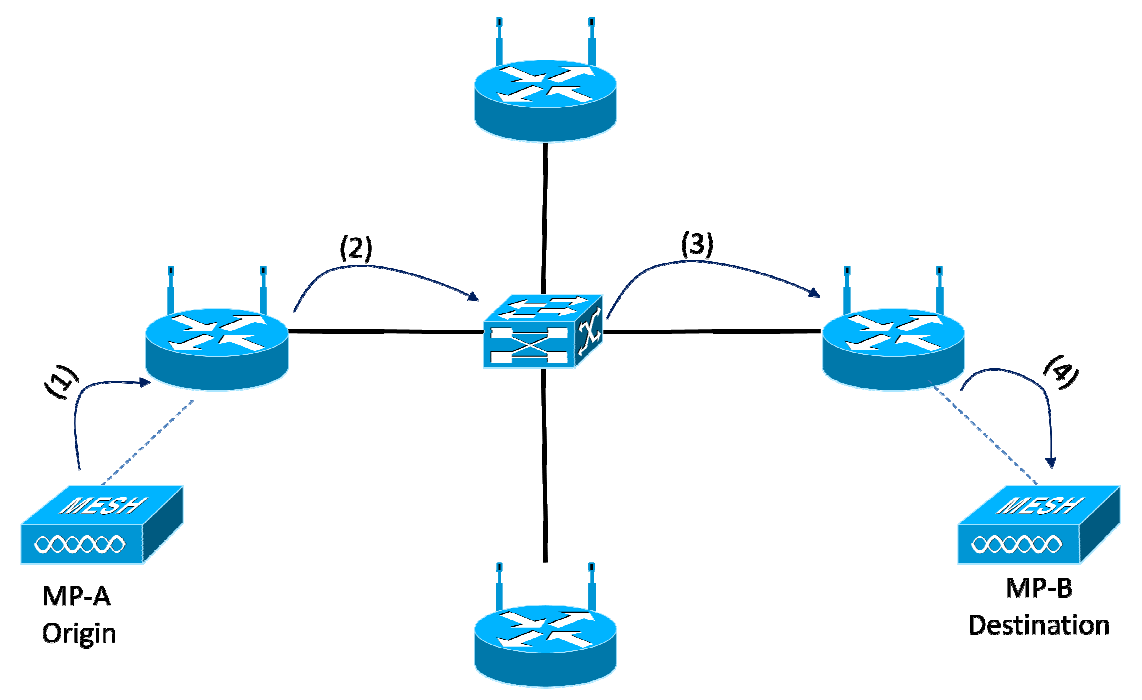

Fig. 2. Illustration of the collaboration of the MPP for the transmission of intra-mesh traffic.

Due to the interworking of the link layer and the Ethernet modules in the MPPs, the number of hops that the packets traverse in the network will be considerably reduced. Besides, the use of the wired links (instead of the wireless mesh links) also avoids packet collisions and minimizes the congestion in the radio channel, which improves the behavior of the mesh communications.

On the other hand, the combination of the two interfaces in the MPPs also affects the portal discovery. As previously said, the MPPs are represented by one of the nodes in the EAT. This role rotates between the MPPs periodically. When representing the group, the MPP emits an announcement containing all the MAC addresses of the group. This announcement is transmitted through the two network interfaces in the MPP. Thus it is received by both the wireless mesh nodes as well as by the others wired MPPs. In this way, all the MPPs regularly announce their presence to the two networks. In a similar way, Route Request messages in the mesh network demanding the route to any MP are also derived by the MPPs and propagated through the wired infrastructure. Thus, multihop communications between two mesh nodes can benefit from the wired links.

\section{Evaluation}

The performance of our proposal is evaluated by means of simulations carried out in OMNeT++ [6]. The simulated scenario employs an area representing the Teatinos Campus of the University of Málaga. The campus and, consequently, the simulation area are $1245 \times 630 \mathrm{~m}^{2}$. In order to evaluate our proposal, we have placed one MAP (i.e. nodes giving access to the mesh network) in every place where the access to the Internet is expected to be more demanded, that is, in libraries, exposition halls and 
cafés. This has led to a network composed of 77 MAPs. In addition, 5 connected MPPs are also incorporated in the mesh architecture in order to enable the Internet connection. Four of these 5 MPPs are placed in the corners of the campus area while another one is located in the center of the simulation area. The 5 MPPs are wired connected by means of a Giga Ethernet network. 40 users (mobile nodes with Wi-Fi interfaces) can access to the network by connecting to a MAP. We have conducted two set of experiments varying the mobility model and the traffic load. Concerning the mobility, the two considered mobility patterns are the model presented in [18] which is an obstacle-based mobility model and the well known Random WayPoint Mobility Model [19]. In both cases, the speed is chosen by means of a Uniform probability distribution from 1 to $2 \mathrm{~m} / \mathrm{s}$ and the pause time is set to 0 seconds. Over 3000 seconds, 12 out of the 40 mobile nodes perform as traffic sources. The conducted experiments include some tests where only Internet traffic is present and some other tests that also incorporate intra-mesh traffic (i.e.: traffic between users). In this last case, $50 \%$ of the injected uplink traffic is for another MP in the network while the resting $50 \%$ of the uplink traffic is routed through the Internet (a MPP is needed). In all the experiments, downlink and uplink traffic loads are equivalent. In this way, the MPPs inject the data transmitted from external Internet hosts. The transmission rate is varied in our experiments to analyze the performance of the proposal. For every transmission rate, 5 simulation runs with different seeds have been executed.

This work aims at evaluating a simplified $802.11 \mathrm{~s}$ based network. Consequently, the routing protocol supporting the communication is AODV, which is the reactive scheme included in the HWMP [5]. Concerning the propagation model, a Two Ray has been selected. The antennas of the MPs are assumed to be at a height of 1 meter. The transmission range is fixed to 100 meters. Other simulation parameters are summarized in Table 1.

Table 1. Simulation parameters.

\begin{tabular}{ll}
\hline Simulation parameters & Value \\
\hline $\begin{array}{l}\text { Simulation area } \\
\text { Simulation time }\end{array}$ & $1245 \times 630 \mathrm{~m}^{2}$ \\
$\begin{array}{l}\text { Number of experiments for a } \\
\text { transmission rate }\end{array}$ & 5 \\
Number of MPPs & 5 \\
Number of MAPs & 77 \\
Number of mobility nodes & 40 \\
Number of sources & 12 \\
Period of the MPP announcements & $100 \mathrm{~s}$ \\
Propagation model & Two Ray \\
Coverage area & $100 \mathrm{~m}$ \\
Ethernet connection between MPPs & 10 Giga Ethernet
\end{tabular}


The network performance is quantified with two metrics: the frame delivery ratio and the end-to-end delay (delay in the section of the routes within Internet is not emulated). In the first set of experiments, we aim at evaluating the impact on the performance when using the anycast discovery and transmission, which are two of three fundamental features of our proposal. By exclusively generating Internet traffic, the redirection of the routing information (Route requests) through the MPPs does not affect this analysis. The mobility model used in these tests is the Random WayPoint. The results are represented in Fig. 3 and Fig. 4. The results represent the mean value of the measured metric and the associated $95 \%$ confidence interval.

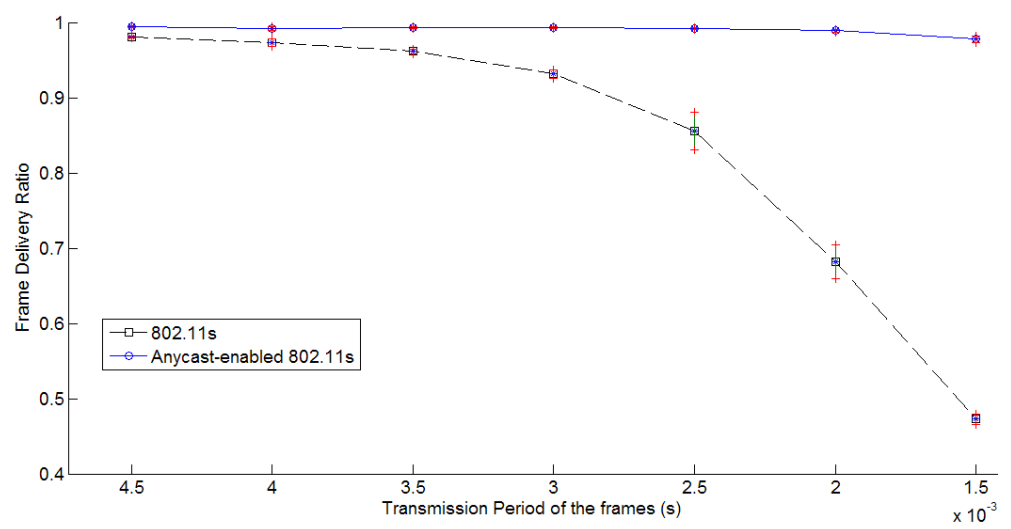

Fig. 3. Frame delivery ratio versus the transmission period for Internet traffic.

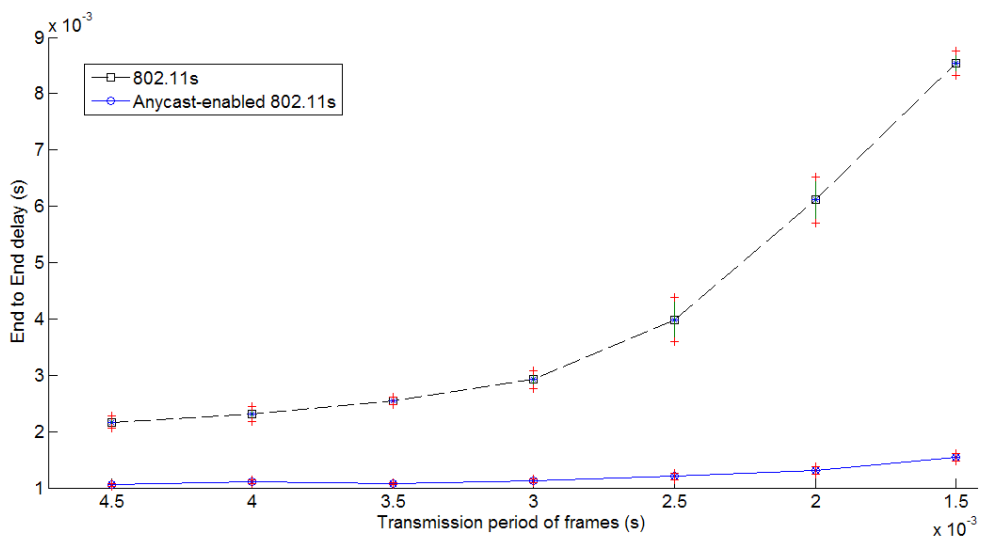

Fig. 4. End-to-End delay versus the transmission period for Internet traffic.

As it can be observed, the frame delivery ratio clearly improves when the anycastbased scheme is used. The use of the MPPs as an anycast group can support high transmission rates without perceiving any significant degradation neither in the frame delivery ratio nor in the delay. On the other hand, the benefits of combining the routing information in the MPPs are evaluated by a second set of experiments. In these experiments, we divide the traffic generated by the MPs into intra-mesh traffic and Internet-traffic. The proportion of the traffic load injected by each type is 
equivalent. Fig. 5 and Fig. 6 show the frame delivery ratio and the delay obtained under these circumstances. The use of this capability reduces the number of hops that the frames traverse. The reduction also implies a clear decrement in the wireless interferences. In turn, the frame delivery ratio increases (up to practically $100 \%$ ). On the other hand, the route lengths are also decremented. Consequently, the delay diminishes.

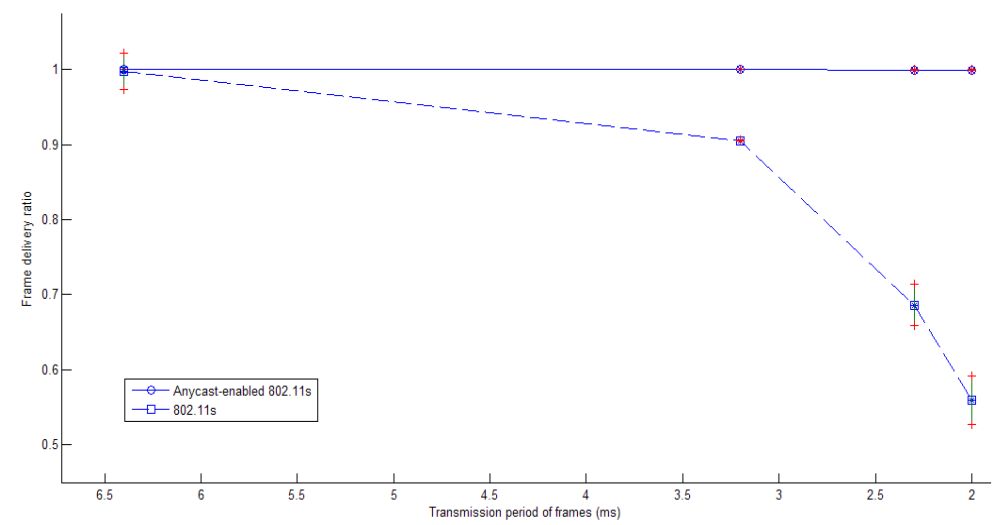

Fig. 5. Frame delivery ratio versus the transmission period for Internet and intra-mesh traffic.

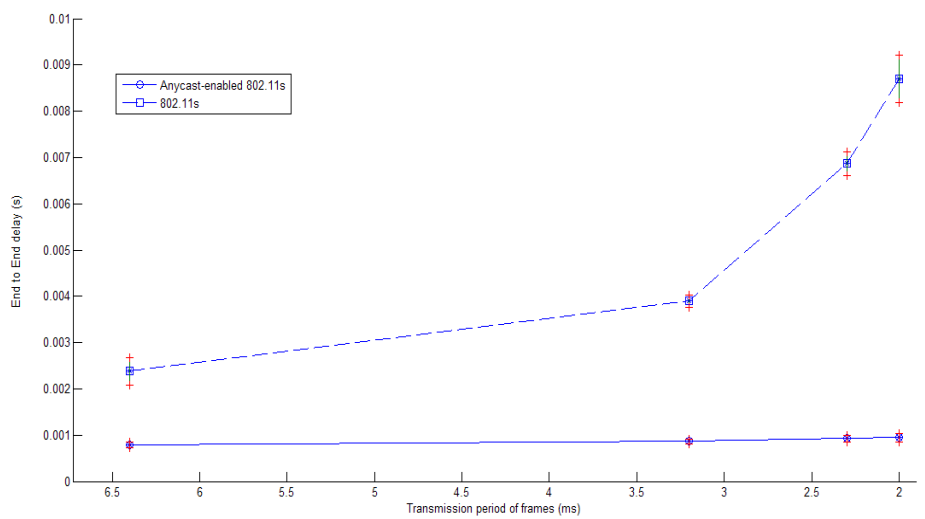

Fig. 6. End-to-End delay versus the transmission period for Internet and intra-mesh traffic.

\section{Conclusions}

This paper has presented a mechanism by which anycast-type transmission is enabled in $802.11 \mathrm{~s}$ mesh networks. The anycast transmission is employed to communicate with the external hosts through an MPP (Mesh Portal). By means of the anycast implementation, a dynamic selection of the MPP can be performed. Additional, the 
two radio interfaces in the MPP are combined so that the intra-mesh traffic can be diverted and supported by the wired connections between the MPPs. The proposal allows the use of customized communication costs and it can be also applied in other link-layer based networks. The simulation results show that the frame delivery ratio is clearly improved while the end-to-end delay is reduced.

\section{Acknowledgment}

This work has been partially supported by the National Project No. TEC200913763-C02-01.

\section{References}

1. Aguayo, D., Bicket, J., Biswas, S., Morris, R.: RoofNet, experimental mesh network, http://pdos.csail.mit.edu/roofnet/doku.php

2 Freifunk, International Project for free wireless networks and frequencies http://start.freifunk.net/

3 802.11s IEEE Draft Standard for Information Technology-Telecommunications and information exchange between systems-Local and metropolitan area networks-Specific requirements-Part 11: Wireless LAN Medium Access Control (MAC) and Physical Layer (PHY) specifications-Amendment 10: Mesh Networking

4 Hiertz, G.R., Denteneer, D., Max, S., Taori, R., Cardona, J., Berlemann, L., Walke, B.: IEEE 802.11s: The WLAN Mesh Standard, IEEE Wireless Communications, vol. 17, pp. 104-11 (2010)

5 Bahr, M.: Proposed Routing for IEEE 802.11s WLAN Mesh Networks. In: $2^{\text {nd }}$ Annual International Wireless Internet Conference (WICON), ACM, Boston (2006)

6 Varga, A.:OMNeT++ User Manual, http://www.omnetpp.org/

7 Pal, A., Nasipuri, A.: GSQAR: A Quality Aware Anycast Routing Protocol for Wireless Mesh Networks. In: IEEE Global Communications Conference (Globecom), pp. 1-5, Miami (2010)

8 Lakshmanan, S., Sivakumar, R., Sundaresan, K.: Multi-gateway association in wireless mesh networks, Ad Hoc Networks, vol. 7, Issue 3, pp. 622-637 (2009)

9 Ashraf, U., Abdellatif, S., Juanole, G.: Gateway Selection in Backbone Wireless Mesh Networks. In: IEEE conference on Wireless Communications \& Networking Conference (WCNC), Budapest (2009)

10 Isabwe, G. M. N., Kim, K.-S.: A Novel Approach to WLAN Mesh Interworking with Multiple Mesh Portals. In: 5th IEEE International Conference on Mobile Ad Hoc and Sensor Systems (MASS), 641 - 646, Atlanta (2008).

11 Sharif, K.; Cao; L., Wang, Y., Dahlberg, T.: A Hybrid Anycast Routing Protocol for Load Balancing in Heterogeneous Access Networks. In: 17th International Conference on Computer Communications and Networks (ICCCN), pp. 1-6, St. Tomas (2008)

$12 \mathrm{Hu}$, Y., He., W, Yang, S., Zhou, Y.: Multi-Gateway Multi-Path Routing Protocol for 802.11s WMN. In: IEEE 6th International Conference on Wireless and Mobile Computing, Networking and Communications (WiMob), pp. 308-315, Niagara Falls (2010)

13 Ben-Othman, J., Mokdad, L., Cheikh, M. O.: Q-HWMP: improving end-to-end QoS for 802.11s based mesh networks. In: IEEE Global Communications Conference (Globecom), Miami (2010) 
14 The One Laptop per Child project, http://laptop.org/en/

15 Ancillotti, E., Bruno, R., Conti, M.: Load-balanced routing and gateway selection in wireless mesh networks: Design, implementation and experimentation. In: IEEE International Symposium on A World of Wireless, Mobile and Multimedia Networks (WoWMoM), Montreal (2010)

16 Song, W., Fang, X-M.: Design and Simulation of Fairness-aware Routing Algorithm in Wireless Mesh Networks, System Simulation, pp. 4320-4325 (2007)

17 Papadaki, K., Friderikos, V.: Joint Routing and Gateway Selection in Wireless Mesh Networks. In: IEEE conference on Wireless Communications \& Networking Conference (WCNC), pp. 2325, Las Vegas (2008)

18Triviño-Cabrera, A., Morales-Berrocal, R., Casilari, E.: Simulation of realistic mobility patterns for mobile ad hoc networks. In: 7th Conference on 7th WSEAS International Conference on Applied Computer Science, pp. 227-231, Venice (2007)

19 Yoon, J., Liu, M., Noble, B.: Random WayPoint considered harmful. In: 22nd Annual Joint Conference of the IEEE Computer and Communications Societies (INFOCOM), pp. 13121321, San Francisco (2003) 